\title{
Visualization of fast-moving cells in vivo using digital holographic video microscopy
}

\author{
Hongyue Sun \\ University of Aberdeen \\ School of Engineering \\ Aberdeen AB24 3UE, Scotland \\ United Kingdom
}

\section{Bing Song}

University of Aberdeen

School of Medical Sciences

Aberdeen AB25 2ZD, Scotland

United Kingdom

\section{Hongpai Dong}

University of Aberdeen

School of Engineering

Aberdeen AB24 3UE, Scotland

United Kingdom

\section{Brian Reid}

University of Aberdeen

School of Medical Sciences

Aberdeen AB25 2ZD, Scotland

United Kingdom

\author{
Michael A. Player \\ John Watson \\ University of Aberdeen \\ School of Engineering \\ Aberdeen AB24 3UE, Scotland \\ United Kingdom
}

\author{
Min Zhao \\ University of Aberdeen \\ School of Medical Sciences \\ Aberdeen AB25 2ZD, Scotland \\ United Kingdom \\ and \\ University of California, Davis \\ School of Medicine \\ Department of Dermatology \\ 1515 Newton Court \\ Davis, California 95616-4859 \\ E-mail:minzhao@ucdavis.edu
}

\begin{abstract}
Digital in-line holography offers some significant advantages over conventional optical holography and microscopy to image biological specimens. By combining holography with digital video microscopy, an in-line holographic video microscope is developed and is capable of recording spatial 3D holographic images of biological specimens, while preserving the time dimension. The system enables high-speed video recording of fast cell movement, such as the rapid movement of blood cells in the blood stream in vivo. This capability is demonstrated with observations of fast 3-D movement of live cells in suspension cultures in response to a gentle shake to the Petri dish. The experimental and numerical procedures are incorporated with a fast reconstruction algorithm for reconstruction of holographic video frames at various planes ( $z$ axis) from the hologram and along the time axis. The current system enables both lateral and longitudinal resolutions down to a few micrometers. Postreconstruction processing of background subtraction is utilized to eliminate noise caused by scattered light, thereby enabling visualization of, for example, blood streams of live Xenopos tadpoles. The combination of digital holography and microscopy offers unique advantages for imaging of fast moving cells and other biological particles in three dimensions in vivo with high spatial and temporal resolution. $\odot 2008$ Society of Photo-Optical Instrumentation Engineers. [DOI: 10.1117/1.2841050]
\end{abstract}

Keywords: holography applications; biomedical optics; microscopy; digital imaging; cells.

Paper 07146RR received Apr. 20, 2007; revised manuscript received Oct. 10, 2007 ; accepted for publication Oct. 12, 2007; published online Feb. 19, 2008.

\section{Introduction}

Recently, significant advances have been made in the technologies for obtaining three-dimensional (3-D) information from imaging biological specimens in two dimensions. Most cell behavioral studies have been carried out with in vitro two-dimensional (2-D) culture models with only a few providing 3 -D information. It becomes more and more obvious

Address all correspondence to: Professor M. Zhao, University of California, School of Medicine, Dermatology, 1515 Newton Ct., Davis CA 95618-4859; Tel: 530-754-5129; Fax: 530-754-5316; E-mail: minzhao@ucdavis.edu that in some instances cellular behavior in three dimensions may be significantly different from that in 2-D cultures. ${ }^{1}$ Various methods have been used to observe and record cell behavior in three dimensions. For instance, migration of greenfluorescence-protein-labeled cells has been studied ${ }^{2}$ in vivo and tumor cell motility in 3-D matrices that involve "rho signaling" from that in 2-D cultures. ${ }^{3,4}$ Using a Hoffman microscope, the in situ behavior of cornea cells is demonstrated. ${ }^{5,6}$ However, only relatively slow cell migration can be imaged

1083-3668/2008/13(1)/014007/9/\$25.00 @ 2008 SPIE 
by these methods and they are generally unsuited to study fast moving cells such as those in blood streams. Although the latter have been studied with other imaging systems, for instance, using a laser scanning ophthalmoscope, ${ }^{7}$ 3-D spatial information was not obtained. When cells move out of the focal plane with high speed, it is impossible to trace them with currently available imaging techniques.

Holography provides biomedical scientists with a unique tool for imaging and analyzing the behavior of living cells in three dimensions in situ. A hologram records the full 3-D information of a scene while retaining the full parallax and perspective of the original scene. It does this on a single planar (2-D) recording medium such as photographic film or an electronic photosensor. The technique is especially attractive for 3-D particle tracking because a 3-D spatial volume can be reconstructed from a single 2-D hologram. If the recording is made using digital techniques, i.e., by recording on an electronic sensor, such as a CCD (charged-coupled device) or CMOS (complementary metal oxide semiconductor) device, and reconstructed numerically within a computer, then holographic videos can be made, thereby retaining the fourth dimension, namely, time. Digital holographic microscopy has a great potential for fast 3-D image acquisition. This has been demonstrated experimentally in a number of papers. For example, in-line digital holography has been used to visualize particle fields $^{8-11}$ in aerosols and biological samples or as a noninvasive contrast imaging tool for observing marine biological samples in 3-D space. ${ }^{12-14}$

Recording holograms on electronic sensors, however, limits the resolution attainable to around 6 to $10 \mu \mathrm{m}$, depending on the pixel size of the sensor, whereas on photographic film about $2 \mu \mathrm{m}$ is possible under favorable conditions. The limitation of spatial resolution can be overcome, to a certain extent, by using a divergent laser beam to illuminate the sample volume and record the hologram on a lensless camera. ${ }^{15}$ Since the depth of field of conventional microscopy is very limited due to the high numerical aperture necessary for high resolution, digital holographic microscopy (DHM) offers the possibility of focusing on different object layers by numerical reconstruction methods while still preserving high resolution. This technique also proved to be successful for 4-D (space and time) tracking ${ }^{16,17}$ of microstructures and organisms using a lensless microscopic system. Algae motion is tracked in a $3-\mathrm{D}$ space along the time coordinate by applying prereconstruction processing, i.e., the sum of subtracted holograms of each of two consecutive video frames. This was successful when dealing with suspended particles with low population density that were moving slowly. Several recent studies ${ }^{18-20}$ utilized a Mach-Zehnder interferometer configuration (with a separated reference beam) with a coherent or partially coherent light source to record intensity and phase-contrast digital microscopic holograms of a 3-D object on a glass slide. The authors successfully demonstrated the ability of DHM for resolving a microstructure of the phase object at high spatial resolution, often down to hundreds of nanometers. Sheng et al. ${ }^{21}$ successfully increased the depth of field and imaging resolution by combining holography with microscopy. This study mainly focused on examining the spatial distribution and velocity of a dense cloud of particles with an extended depth. Tracking cell movement with high speed in three dimensions in vivo has, however, not been attempted.
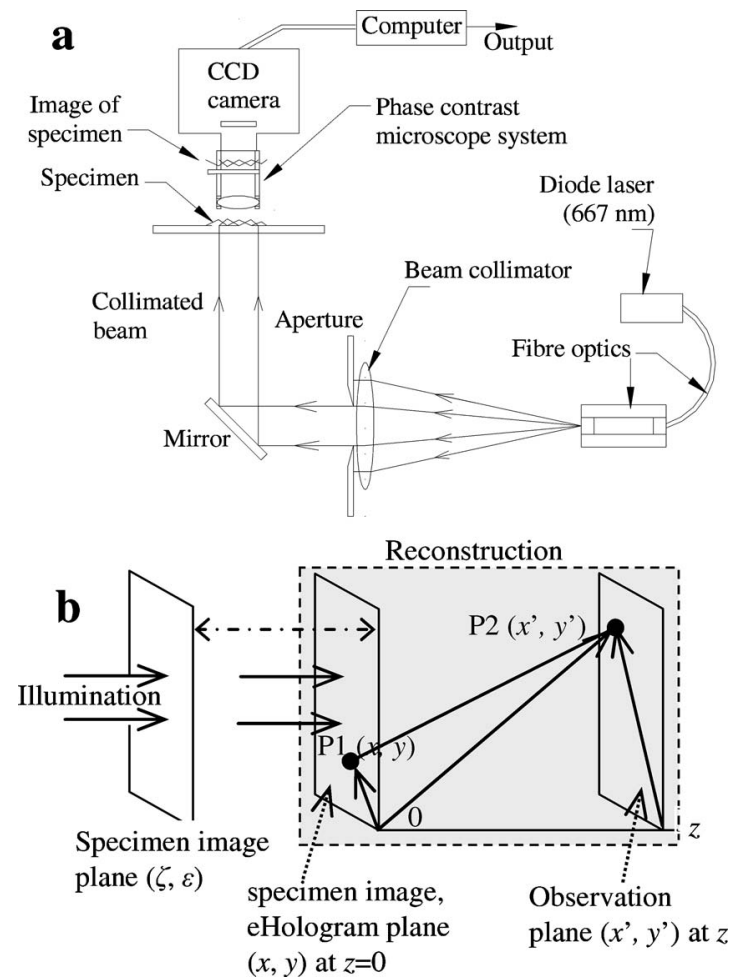

Fig. 1 Schematics of the DHVM system: (a) the DHVM system and (b) reconstruction scheme of an ehologram.

To observe fast-moving cells in 3-D space in animal tissues in vivo, we introduce a digital holographic video microscope (DHVM) with the use of coherent light. This achieves holographic imaging of biological specimens in vitro and in vivo over the 3 -D volumetric space and in time. In this paper, we describe our DHVM system and demonstrate cell motions in different media. We also demonstrate that fast cell motion in blood streams in vivo can be recorded, visualized, and quantified with our system. The system enables both lateral and depth resolutions at the micrometer level. The experimental and numerical procedures have been incorporated into a program package with a fast reconstruction algorithm, which is capable of reconstructing of holographic video frames at various planes ( $z$ axis) from the hologram and in the time dimension after image acquisition. This capability is interpreted in 3-D mappings, showing the motion of live cells (neutrophils) in suspension cultures and fast movement of blood cells in 3-D blood vessels of a live Xenopos tadpole. We also apply postreconstruction processing of background subtraction to eliminate background noise caused by the scattered light from dense cell population density in live tadpole tissue.

\section{Principle and Implementation of DHVM}

A digital/electronic hologram (ehologram) is formed by the interference between waves scattered from an object on coherent illumination and the straight-through wave at the hologram/sensor plane. We took a simplified technical approach to buildup our system. The system [Fig. 1(a)] utilizes a single-mode diode laser (continuous wave, 667-nm wavelength, $10-\mathrm{mW}$ power) that delivers the collimated laser beam to illuminate the specimen through an optical fiber attached 
with a collimator. The microscope generates a magnified real image of the specimen in the space in front of a CCD (Sony ICX-082AL-6) sensor implemented in a Hitachi camera (KP-MA type) together with an associated image grabber (ViewCast Corporation Osprey 210). The sensor dimensions are $8.72 \mathrm{~mm}$ (horizontal) by 6.52 (vertical) $\mathrm{mm}$ with the pixel pitch of $11.6 \mu \mathrm{m}$ (horizontal) by $11.2 \mu \mathrm{m}$ (vertical). The magnified real image and the directly transmitted light through the specimen form an interference pattern in space, i.e., a hologram. So a hologram is observed by varying the observation plane from the in-focus image plane. The interference pattern will then be captured by the CCD imaging sensor at the sensor or hologram plane $(x, y)$. Such a recorded 2-D ehologram, represented by $E(x, y, 0)$, where $(x, y)$ are the position coordinates of an arbitrary point $\mathrm{P} 1$ at the ehologram plane [Fig. 1(b)], stores the interfering fringe patterns and is postprocessed digitally in a computer. The CCD sensor records the 2-D spatial sampling on the recorded ehologram, which can be implemented for numerical reconstruction.

Digital hologram reconstruction has been widely implemented recently in a diverse range of scientific and engineering problems. Reconstructing an ehologram involves tracing the beam propagation through the system and calculating the diffraction pattern by means of a numerical representation of the physical process of diffraction. Many algorithms have been employed based on, for example, the Fresnel transform, ${ }^{22-24}$ the Fraunhofer transform, ${ }^{25}$ the wavelet transform, ${ }^{26}$ and "Fresnelets" with wavelet bases. ${ }^{27}$ The reconstruction algorithm used in our system is derived from the angular spectrum theory. ${ }^{28} \mathrm{~A}$ parallel reconstruction wave propagates through the $2-\mathrm{D}$ ehologram aperture at $z=0$ [Fig. $1(\mathrm{~b})]$, the wave is diffracted by the fringe pattern on the ehologram to form the reconstructed field at the observation plane $\left(x^{\prime}, y^{\prime}\right)$. The reconstructed field at an arbitrary point P2 in $\left(x^{\prime}, y^{\prime}\right)$ plane is $E\left(x^{\prime}, y^{\prime}, z\right)$. The mathematical representation for 3-D diffraction of the angular spectrum is

$$
E\left(x^{\prime}, y^{\prime}, z\right)=F^{-1}\left(F_{h}(x, y, 0) \exp \left\{i z k\left[1-(q / k)^{2}\right]^{1 / 2}\right\}\right),
$$

where $q=q_{x}^{2}+q_{y}^{2}$ is the 2-D variable in the Fourier domain and the conjugate of the variable $\left(x^{\prime}, y^{\prime}\right)$ in the spatial domain, $E\left(x^{\prime}, y^{\prime}, z\right)$ is the reconstructed complex amplitude of the diffraction wavefront in the $\left(x^{\prime}, y^{\prime}\right)$ plane at a $z$ distance from the hologram plane. Here $F_{h}(x, y, 0)$ represents the Fourier transform $(\mathrm{FT})$ of the recorded wave interfering distribution at the hologram plane $(x, y)$ at a point $\mathrm{P} 1, k=2 \pi / \lambda$, and $\lambda$ the wavelength of the illuminating light. This is then rewritten in its integral form after taking into account the Fresnel region approximation $^{28}$ as

$$
\begin{aligned}
E\left(x^{\prime}, y^{\prime}, z\right)= & -\frac{i}{\lambda z} \exp \left(i \frac{2 \pi}{\lambda} z\right) \\
& \times \iint E(x, y, 0) \exp \left\{i \frac { \pi } { \lambda z } \left[\left(x^{\prime}-x\right)^{2}\right.\right. \\
& \left.\left.+\left(y^{\prime}-y\right)^{2}\right]\right\} \mathrm{d} x \mathrm{~d} y .
\end{aligned}
$$

Equation (1) is rearranged as a 2-D convolution:

$$
E\left(x^{\prime}, y^{\prime}, z\right)=E\left(x^{\prime}, y^{\prime}, 0\right) \otimes \frac{-i}{\pi \tau^{2}} \exp \left[i 2\left(\frac{\pi \tau}{\lambda}\right)^{2}\right] K\left(x_{\tau}^{\prime}, y_{\tau}^{\prime}\right)
$$

where $x_{\tau}^{\prime}=x^{\prime} / \tau ; y_{\tau}^{\prime}=y^{\prime} / \tau ; \tau^{2}=\lambda z / \pi$, as the scaled distance parameter; and $K\left(x_{\tau}^{\prime}, y_{\tau}^{\prime}\right)=\exp \left[i\left(x_{\tau}^{\prime 2}+y_{\tau}^{\prime 2}\right)\right]$ is the chirp function and is considered as the mother wavelet. Function $K\left(x_{\tau}^{\prime}, y_{\tau}^{\prime}\right)$ in Eq. (2) is not limited in both spatial and frequency domains, it has the scale and shift features of a wavelet family for 2-D function spaces. Because reconstruction involves numerical convolution of the kernel with the hologram intensity, it is necessary to avoid aliasing in the sampled version of the kernel. To satisfy the wavelet admissibility condition a Gaussian envelope function $G\left(x^{\prime}, y^{\prime}\right)=\exp \left[-\left(x^{\prime 2}\right.\right.$ $\left.\left.+y^{\prime 2}\right) / \delta\right]^{2}$ was introduced by Buraga-Lefebvre et al. ${ }^{29}$ to the mother wavelet. Thus, the Fresnel transform in Eq. (1) can be expressed by a wavelet transform:

$$
E\left(x^{\prime}, y^{\prime}, z\right)=E\left(x^{\prime}, y^{\prime}, 0\right) \otimes k(\tau) K(\tau, p, q),
$$

where $k(\tau)=\left(1 / \pi \tau^{2}\right) \exp \left\{i\left[2(\pi \tau / \lambda)^{2}-(\pi / 2)\right]\right\}$ is the amplitude of the daughter function, $K(\tau, p, q)=K\left[\left(x^{\prime}-x\right) / \tau,\left(y^{\prime}\right.\right.$ $-y) / \tau] G\left(x^{\prime}, y^{\prime}\right)$ is the wavelet set; and $p=\left(x^{\prime}-x\right) / \tau$ and $q$ $=\left(y^{\prime}-y\right) / \tau$. In our DHVM system, the reconstruction algorithm is based on the wavelet transform in Eq. (3). Its kernel is based on that used by Onural and Kocatepe. ${ }^{30}$ However, we adjusted the Gaussian envelope function to provide the necessary filtering, by taking $\delta=\pi \tau / 2 \sqrt{A}$, with a parameter $A$ selected to give a suitable reduction in the envelope amplitude at a value of $\left(x^{\prime 2}+y^{\prime 2}\right)$ such that the wavelet frequency equals the Nyquist frequency. More detailed discussions on the performance of various algorithms can be found from Dong et al. ${ }^{31}$ It is readily verified that for pixel spacing $d$ the envelope amplitude, at the Nyquist frequency, $\delta=\exp \left(-A / d^{2}\right)$, is independent of the reconstruction distance $z$. However, due to the usage of objective lenses through the microscopy system and the in-air wavelength in the reconstruction algorithm, the actual physical distance of the specimen image will not be identical to the reconstruction distance even if a parallel recording beam is used. Therefore, to make a valid measurement of the specimen, it is essential to calibrate the system transversely (along $x^{\prime}$ and $y^{\prime}$ ) and longitudinally (along $z$ ). The transverse scale is calibrated by using a recorded hologram reconstruction of a standard scale slide for microscopy. Similarly, the longitudinal calibration is carried out by recording a hologram of an object with known thickness $\left(d_{g}\right)$ and reconstructing this hologram to find the $z$ interval (in micrometers) of $d_{g}$; in our case, a $0.60-\mathrm{mm}$ glass slide was used. This method will inevitably introduce deviations in $z$ distance calculation, mainly because the refractive index (RI) of the cell solution $\left(n_{c}\right)$ differs from the RI of the glass $\left(n_{g}\right)$, such that in a given time interval, the wave travels different distances in the cell solution $\left(d_{c}\right)$ and the glass. Ideally, the reconstruction program should take into account of the wavelength change when simulating wave propagation in different media. For simplicity, the algorithm presented here uses only one wavelength $(667 \mathrm{~nm})$ to calibrate the longitudinal distance. The inaccuracy $(\Delta d)$ is estimated as less than $10 \%$ of the distance traveled in $d_{c}$, by considering that in a given time interval, the wave optical paths through all media are the same: $d_{g} n_{g}$ 


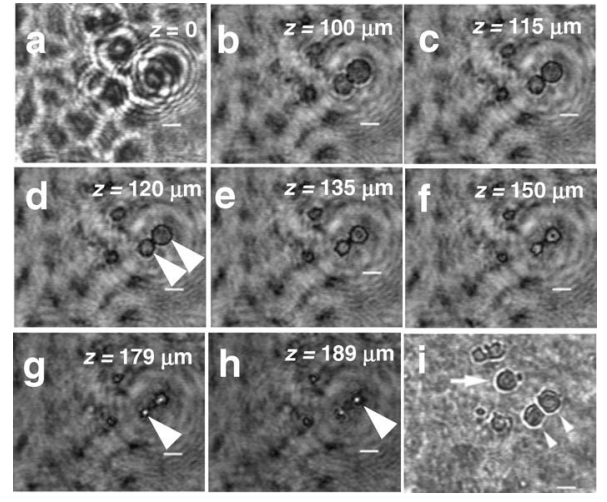

Fig. 2 Trypsinized human umbilical vein endothelial cells in suspension culture visualized by the DHVM: (a) hologram at $z=0$ plane; (b) to (f) reconstructions of (a) at $z=100,115,120$ (two arrowed cells are on focus), 135, and $150 \mu \mathrm{m}$, respectively; (g) and (h) reconstructions of the focal points formed by the arrowed spherical cells in (d); (i) micrograph for the same cells, arrowhead pointing at the on-focus cells; scale bar is $10 \mu \mathrm{m}$.

$=d_{\text {sol }} n_{\text {sol }}$ (e.g., $\left.n_{g} \sim 1.50, n_{c} \sim 1.35, \Delta d / d_{c}=0.1\right)$. The scattering effect was not considered in reconstruction for error estimation because the cell size concerned was larger than the laser wavelength $(667 \mathrm{~nm})$. However, when imaging submicrometer objects and the diffraction limit is reached, care should be taken in configuring the recording system.

\section{Results and Examples}

\subsection{Living Cells in Suspension Culture}

Adherent cells on a planar surface have been successfully imaged with holographic techniques in several laboratories. ${ }^{15}$ In addition to the adherent cells on flat surfaces, with our system cells in suspension can also be recorded. Here we present holograms and reconstructed images of two types of human cells, live umbilical vein endothelial cells and neutrophils, in suspension in culture medium.

Human umbilical vein endothelial cells were collected shortly after trypsin treatment to dislodge them from adhesion. This treatment released the cells from the planar surface into suspension status in a 3-D volumetric space. A typical hologram interference pattern [Fig. 2(a)] and its reconstructed images at different planes demonstrates the viability of such a holographic system in imaging cells in suspension culture, i.e., in a 3-D volume. Figures 2(b) to 2(h) show a sequence of images reconstructed numerically from the hologram [Fig. 2(a), also see Video 1] at reconstruction distances $z$ of 100 , $115,120,135,150,179$, and $189 \mu \mathrm{m}$, respectively [Figs. 2(b)-2(h)], where the two arrowed cells are in focus at $z$ $=120 \mu \mathrm{m}$. A 2-D in-focus micrograph of the same group of cells is presented [Fig. 2(i)] for comparison with the holographic images. Details of cell morphology and contrast in the in-focus holographic image appear comparable to or better than the in-focus micrographic image. Holographic images can be reconstructed at any $z$ plane and offer detailed 3-D volumetric information. We notice that two bright dots, indicated by a white arrow in Figs. 2(g) and 2(h), are present in

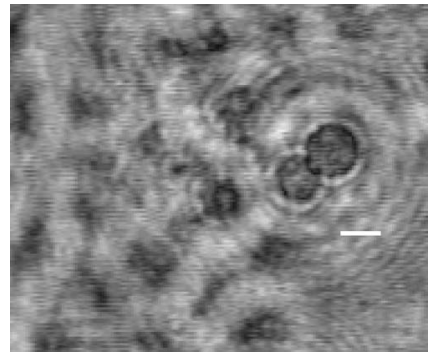

Video 1. Reconstructed hologram sequence of human umbilical vein endothelial cells in $z$ axis. Steps of $z$ 30, step interval; $5.5 \mu \mathrm{m}$; whole frame size, $105 \times 88 \mu \mathrm{m}$ (QuickTime, 3.0 MB).

[URL: http://dx.doi.org/10.1117/1.2841050.1]

the reconstruction domain at $z=179$ and $z=189 \mu \mathrm{m}$. These are formed by the sphere-shaped cells [arrowed in Fig. 2(d), respectively], which act as small positive lenses, focusing the collimated illumination beam on their focal points, i.e., the bright dots. One can accurately measure the distance between the in focus reconstruction image [e.g., Fig. 2(d)] and the bright spots along $z$. The measured distance can be used to obtain cell property information, such as the refractive index of cell (if the cell diameter is known) or the dimension of the cell along $z$ (if its refractive index is known). The distance between the spherical centers of these cells and their focal points [i.e., the effective focal length (EFL) of the cell lens], are obtained: $E F L=29$ and $69 \mu \mathrm{m}$ for the bottom-left and top-right arrowed cells, respectively, in Fig. 2(d).

\subsection{Rapid Cell Motion in 3-D Suspension Culture}

Cells can move rapidly when they are grown in a volume of suspension culture. It is impossible to track many such fastmoving cells simultaneously with conventional microscopy because one can only choose one focal plane at one time point. In DHVM recording, however, it is the interference patterns of all cells in the light path that a hologram stores, so that choosing a focal plane is no longer an issue. The original scenes of moving cells in the recording volume are obtained and extracted to show, for instance, the image focal plane and the 3-D spatial properties in the reconstruction stage. By reconstructing an electronic holographic video (eholovideo) in time sequence, fast-moving cells in a volume can be recorded on an eholovideo and then reconstructed for visualization and quantitative determination of the 3-D distribution over the recording period. To observe cell motion in free space, we chose small nonadherent cell lines (HL60 cells) in suspension culture as our volumetric recording object. HL60 cells are from the promyelocytic leukemia cell line, which normally grow nonadherently in suspension in culture medium. A gentle shake to the Petri dish caused fast movement of the cells in a volume. The movement was recorded in a 3-s eholovideo (original video not shown) using the system shown in Fig. 1(a). The eholovideo was recorded at a rate of $10 \mathrm{fps}$ (frames per second). A reconstruction video at a certain $z$ distance $698 \mu \mathrm{m}$ against the time axis is shown in Video 2. In this reconstruction, one individual cell (arrowed) was shown in focus in some of the video frames at the chosen $z$ distance at various time points, but was not visible on the adjacent reconstructed frames, indicating that cells moved in 


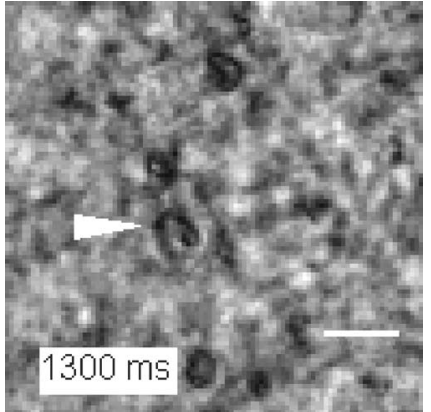

Video 2. Eholovideo (digital holographic video) of fast-moving neutrophil cells. This video is a reconstructed eholovideo from a 3-s eholovideo (not shown) recorded at frame rate of $10 \mathrm{fps}$. The focusing plane $z$ was randomly selected to illustrate the movement of neutrophils in time sequence in 3-D space. One of the in-focus cells, pointed to by an arrowhead, is visible only at some certain time points, e.g., at 400, 1300, and $2300 \mathrm{~ms}$, indicating that cells move in and out of the chosen focusing plane over the time. Neutrophil cells were grown in suspension culture at the time of recording. Scale bar is $50 \mu \mathrm{m}$ (QuickTime 2.5 MB).

[URL: http://dx.doi.org/10.1117/1.2841050.2]

and out of the chosen $z$ plane as well as in the $x^{\prime}$ and $y^{\prime}$ directions (Video 2) along the time axis. By reconstructing each hologram frame in the video in time sequence, the exact locations of moving cells are extracted; therefore, the fast movement of cells in three dimensions can be visualized and quantified successfully. Some eholovideo frames and their reconstructions at the chosen $z$ distance of $698 \mu \mathrm{m}$ are selected to show one individual cell reconstruction at the indicated time points, marked in Figs. 3(a)-3(c), corresponding to the movement of the selected HL60 cell in $3 \mathrm{~s}$, shown in the 3-D mapping in Figs. 3(d)-3(f), respectively. A video of the 3-D trajectory for this selected cell was generated (Video 3) in MATLAB to further illustrate this motion schematically. According to the reconstruction coordinates $(x, y, z)$ at each time point, the motion speed is estimated to be $260 \pm 26 \mu \mathrm{m} \mathrm{s}^{-1}$ on average. Imaging such rapid cell motion is almost impossible with other imaging systems currently available, because the speed at which cells move in and out of the observation plane in a volume is too high to be focused by the imaging system.

\subsection{Spatial Distribution of Cells In Vivo}

Subpopulations of cells in a living organism have a defined spatial distribution. A single hologram contains the 3-D infor-

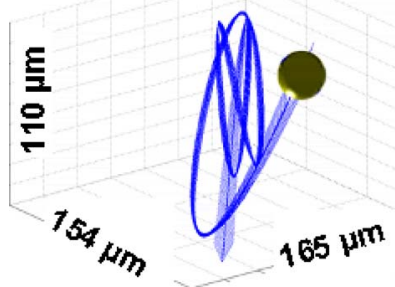

Video 3. Four-dimensional schematic of the 3-D mapping of a fastmoving neutrophil along the fourth dimension of time (QuickTime, 0.7 MB). [URL: http://dx.doi.org/10.1117/1.2841050.3]
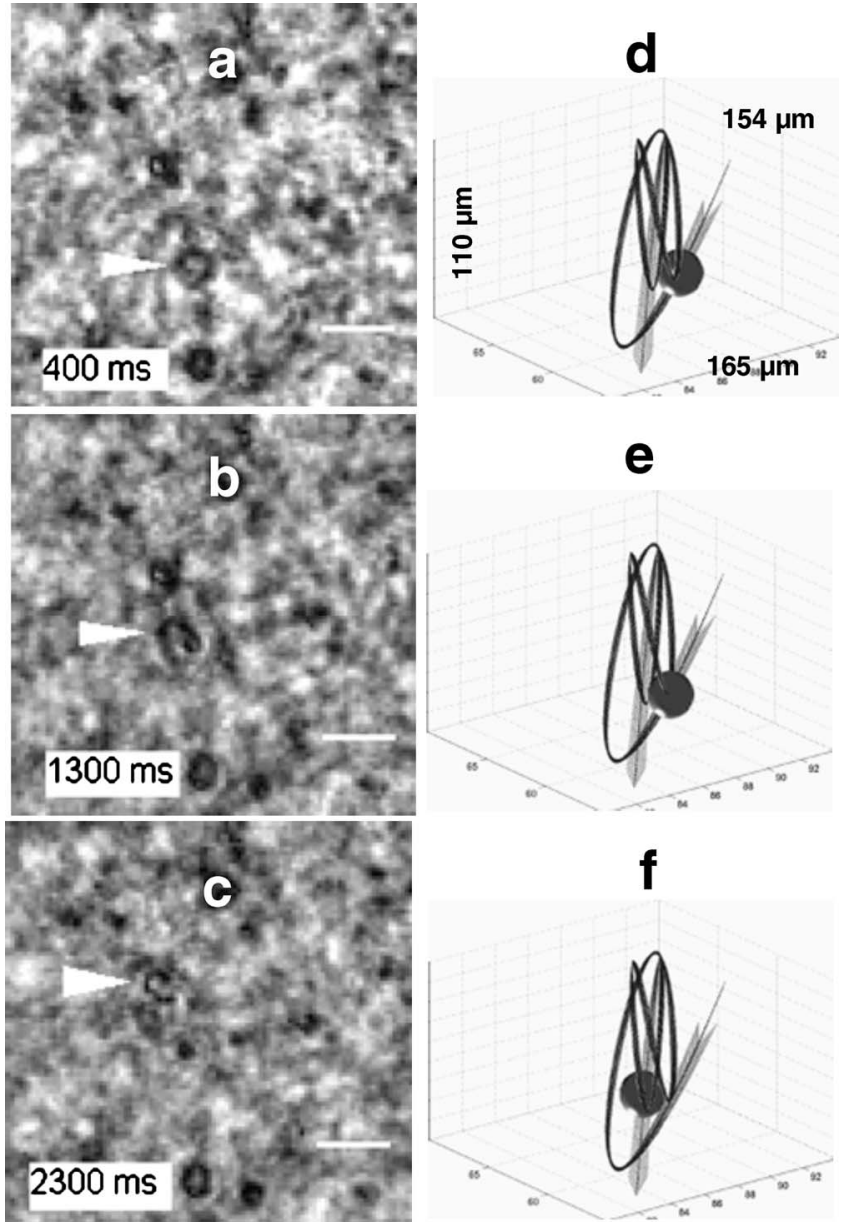

Fig. 3 Fast-moving neutrophils in suspension culture captured and visualized with the DHVM: (a) to (c) the same in-focus cell, pointed to by an arrowhead, at a given $z$ distance but at various time points of 400,1300 , and $2300 \mathrm{~ms}$, respectively; (d) to (f) the corresponding relative locations of the pointed-to cell in (a) to (c) in a 4-D (including time) mapping. Axis dimensions are as indicated in (d) and the scale bar is $20 \mu \mathrm{m}$.

mation that reveals such a spatial distribution of cells at one time point. A large amount of cells in the tail region of a live tadpole are shown in Fig. 4. From a single recorded hologram [Fig. 4(a)], we were able to reconstruct the images and quantify the distribution of cells at the moment that the hologram was taken. Fig. 4(b) and 4(c) show the reconstructions of Xenopus tadpole tail at $z=178$ and $58 \mu \mathrm{m}$ for the cell groups marked in white rectangles, respectively, which were enlarged for the convenience of visualization. The in-focus cell groups are randomly chosen from various focal planes through $z$ and plotted in a 3-D mapping to illustrate their spatial relationship [Fig. 4(d)] and the distribution in a 3-D tissue space at the time point. The arrowhead in Fig. 4(d) points to the cells located in the enlarged area marked as "group b" in Fig. 4(b) at the $z=58 \mu \mathrm{m}$ plane; the arrow points at the cells in one of the enlarged areas marked as "group c" in Fig. 4(c) at the $z$ $=178 \mu \mathrm{m}$ plane. The whole reconstruction through the $z$ axis is given in Video 4, in which one can choose to view the in-focus cells at any arbitrary plane through depths. 


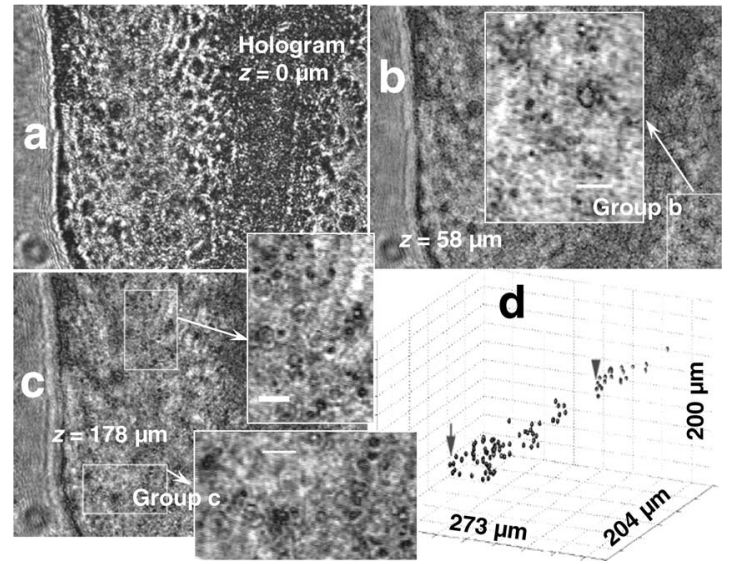

Fig. 4 Three-dimensional distribution of cell population in vivo captured and visualized in a live Xenopus tadpole by a single digital hologram: (a) hologram; (b) in-focus cell group (group b) at the $z$ $=58 \mu \mathrm{m}$ plane; (c) in-focus cell groups (group c) at $z=178 \mu \mathrm{m}$; where the in-focus cell images (in white rectangles) were enlarged to be visualized; (d) 3-D mapping of the in-focus cell groups, with the arrowhead pointing at "group b" cells in (b) and the arrow sign pointing at "group c" cells in (c). Axis dimensions are as indicated and scale bar is $32 \mu \mathrm{m}$.

\subsection{Digital Holographic Video of Fast Cell Movement in Blood Stream In Vivo}

Cell movement in blood streams has been reported with video microcopy for decades. However, when cells move out of the focal plane of the objective lens at a speed such as in the blood stream, conventional video microscopy is not able to capture the movement. With our digital holographic video system, we recorded in vivo blood cells in the tadpole blood stream. Fast-moving cells could be tracked and quantified in three dimensions. A 3-s eholovideo (not shown) of the tadpole tail with blood vessels was recorded, in which two blood stream lines are seen when playing back the video. Figure 5(a) shows the 14th frame $(t=1400 \mathrm{~ms}, z=0)$ where, the two

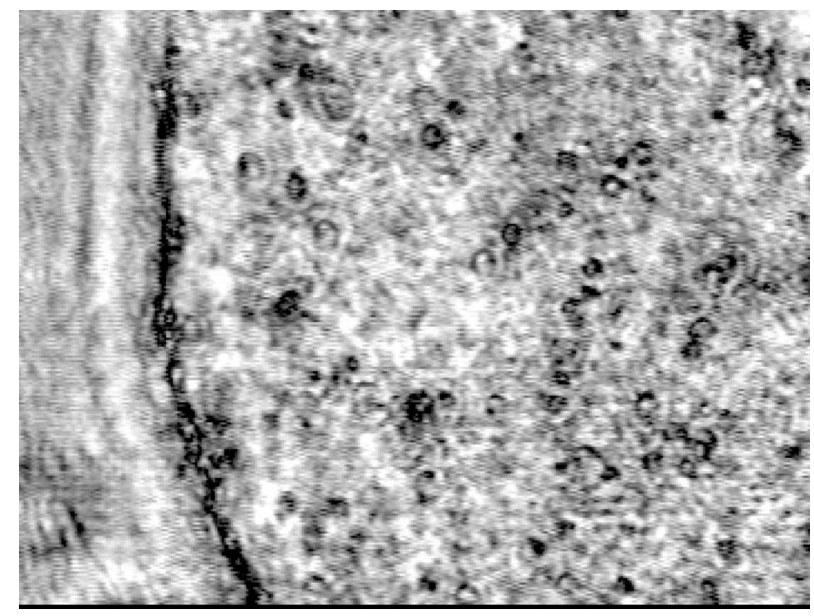

Video 4. Eholovideo reconstruction of a tissue block in vivo. An ehologram (digital hologram) was recorded [Fig. 4(a) in the main text] using Xenopus tadpole tail tissues and reconstructed at various focal planes (31 steps) with a step interval of $5.5 \mu \mathrm{m}$ (QuickTime 1.6 MB). [URL: http://dx.doi.org/10.1117/1.2841050.4]

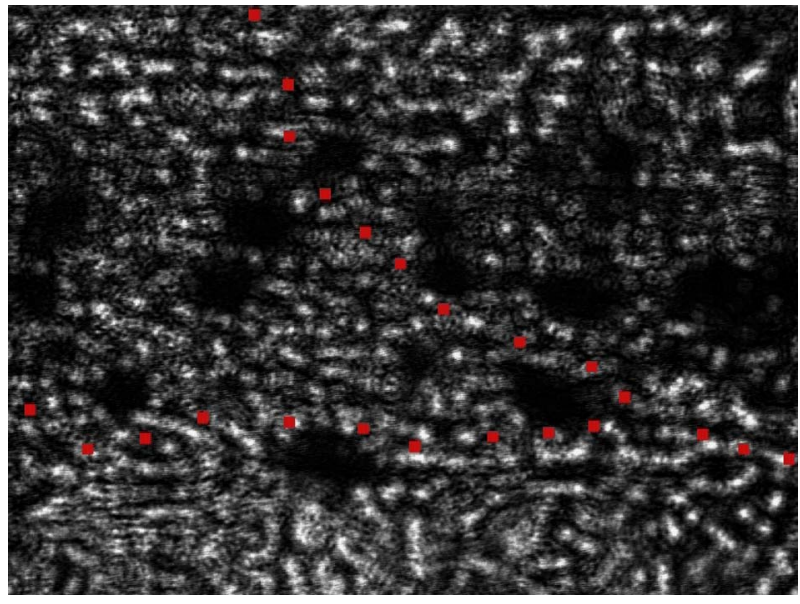

Video 5. Visualization of tadpole blood vessels in vivo. A live tadpole was used to record a 3-s eholovideo (not shown) with $10 \mathrm{fps}$ frame rate. The eholovideo is reconstructed along the time axis at the reconstruction distance $75 \mu \mathrm{m}$. We see that only several blood cells are in focus at this reconstruction distance. The full frame size is 273 (width) $\times 204 \mu \mathrm{m}$ (height) (QuickTime 2.7 MB).

[URL: http://dx.doi.org/10.1117/1.2841050.5]
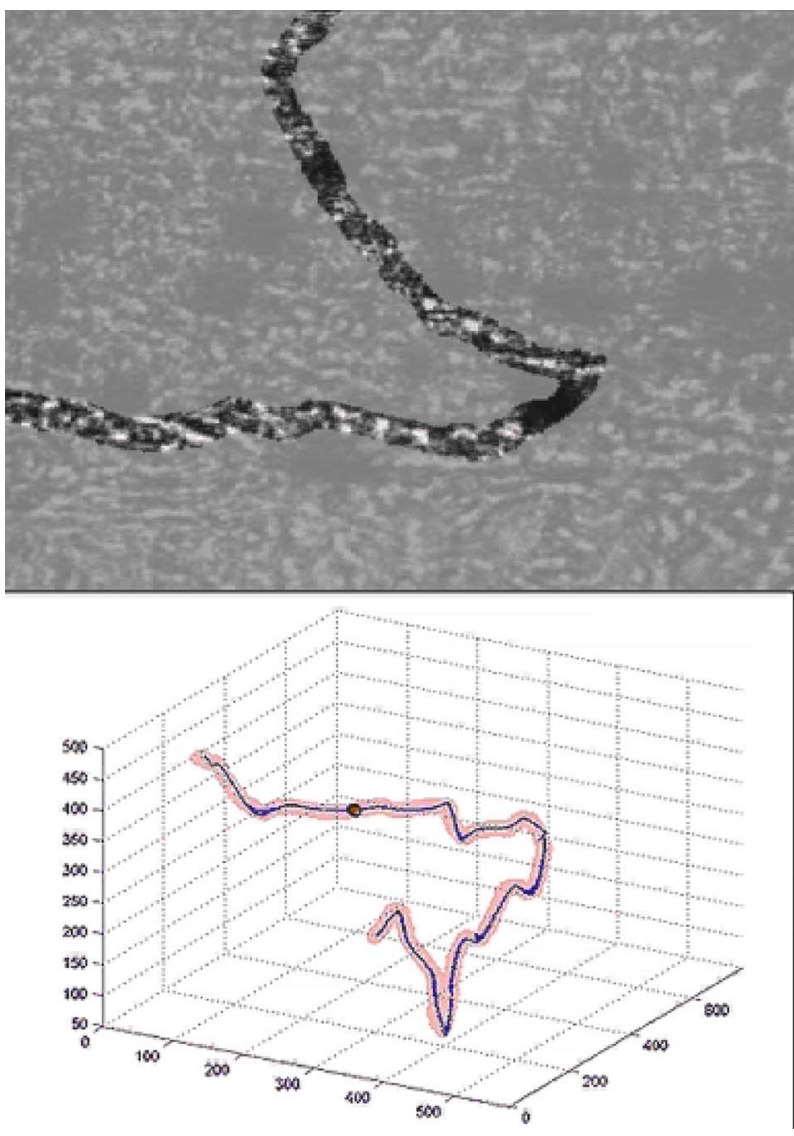

Video 6. Four-dimensional blood cell movement in the tadpole blood vessel. Upper frame, eholovideo of tadpole blood vessel which was recorded at the frame rate of $10 \mathrm{fps}$ over a 3-s period. Individual blood cells are detected to show their migration in the blood vessel along the time axis. The main blood stream, marked as "Main stream" in Fig. 5(a) in the main text, was highlighted as a clear image against a blurred background area. Lower frame, schematic 3-D mapping of the blood cell movement in the "Main stream" (QuickTime 2.1 MB). [URL: http://dx.doi.org/10.1117/1.2841050.6] 

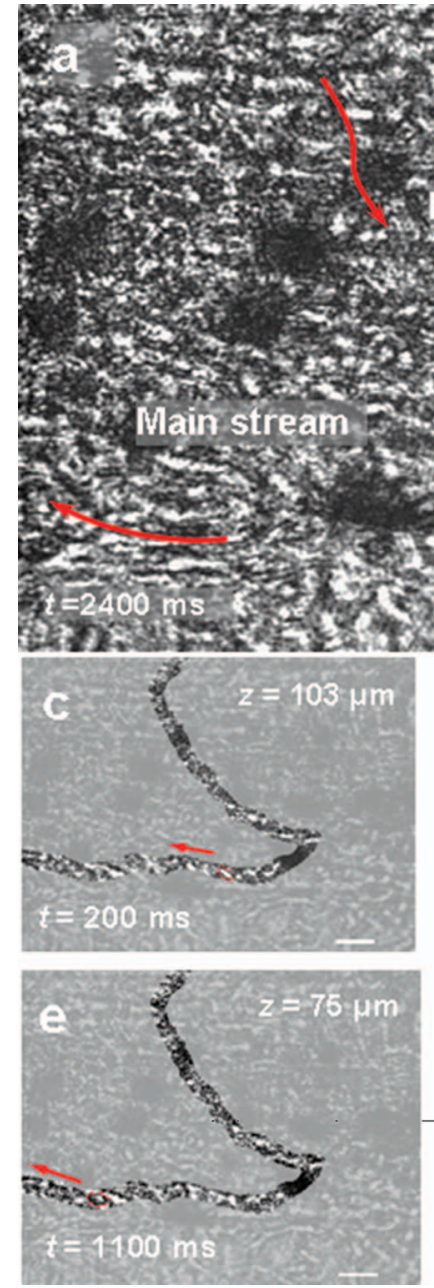

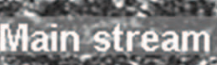

Hain stream
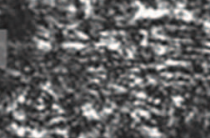

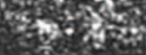
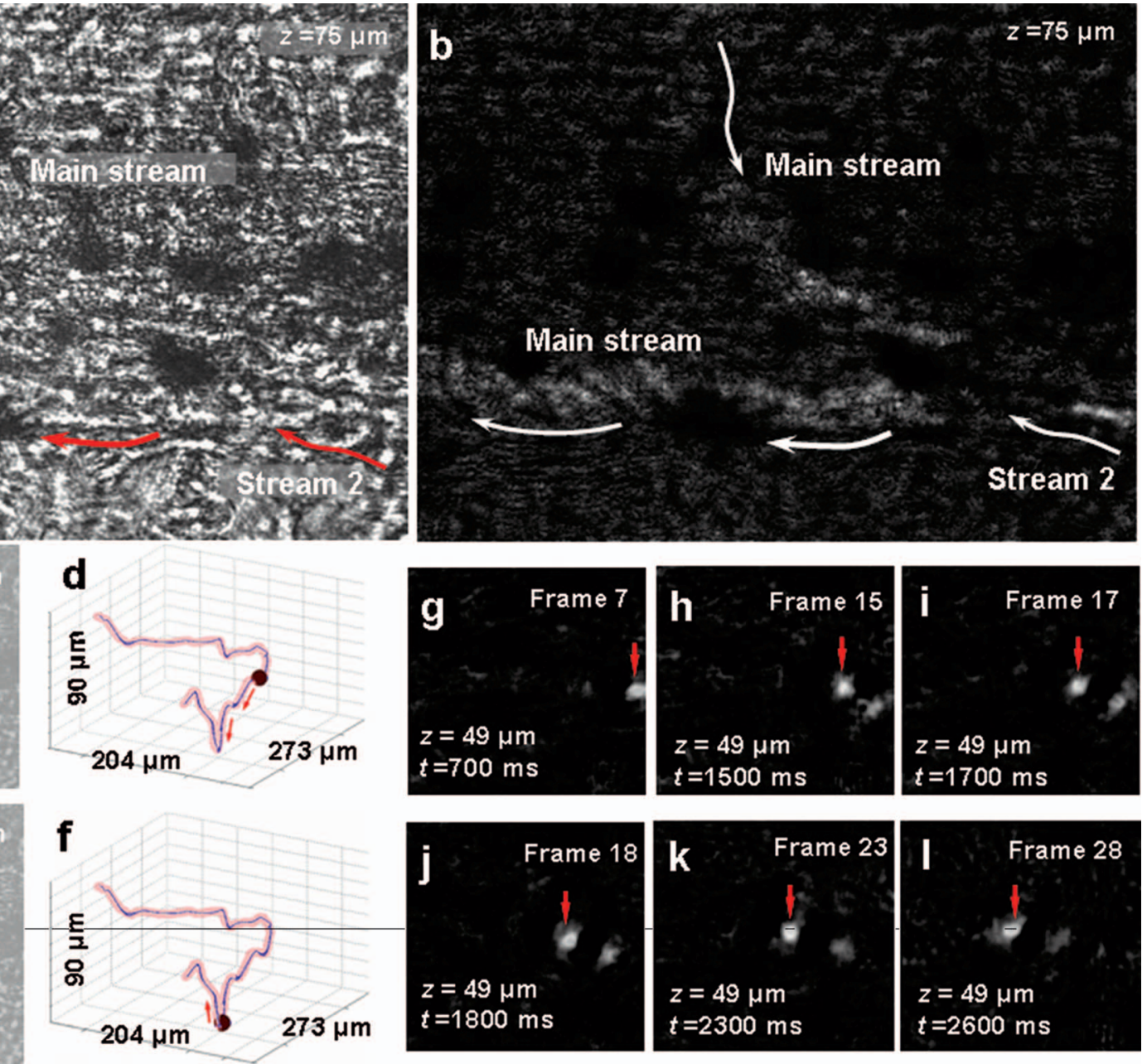

Fig. 5 Visualization of fast-moving cells in blood vessel in vivo using the DHVM: (a) an eholoimage of tadpole tissue, the first frame ( $t=0$ ) from a 3 -s eholovideo recorded with an $\times 32$ lens; (b) the sum of subtracted holograms (at $z=75 \mu \mathrm{m}$ ) of a tadpole blood vessel, tracked two blood streams, i.e., "Main stream" and "Stream 2"; (c) and (e) reconstructed cell (marked in a red circle) in blood stream "Main stream" (highlighted) at time points of 200 and $1100 \mathrm{~ms}$, and at a $z$ distances of (c) 103 and (e) $75 \mu \mathrm{m}$, respectively; (d) and (f) schematic mapping of the tracked cell in a 4-D representation, i.e., 3-D space and time course; the time axis dimension is 3000 ms and the space dimensions are as indicated, the scale bar is $20 \mu \mathrm{m}$; (g) to (I) reconstruction images of an individual cell extracted from Video 8. The individual cell, pointed to and followed by a red arrow, flows in the blood stream "Stream 2," and is reconstructed at a $z=49 \mu \mathrm{m}$ distance and various time points, as indicated in each image. The frame numbers shown are identical to the frame numbers in Video 5 and the whole frame size is 43 (width) $\times 41$ (height) $\mu \mathrm{m}$.

blood streams were marked as "Main stream" and "Stream 2" with red arrows pointing at the blood flow directions. The eholovideo was then reconstructed at $z=57 \mu \mathrm{m}$ against the time axis (Video 5). The spatial distribution of blood cells in the "Main stream" is tracked by reconstructing holograms at each time point $(t)$ and scanning through the $z$ axis in a range of 30 to $120 \mu \mathrm{m}$. Fig. 5(c) and 5(e) illustrate two examples of such tracked blood cell, marked with a red dashed circle, at $z=103 \mu \mathrm{m}$ and $t=200 \mathrm{~ms}$ and $z=75 \mu \mathrm{m}$ and $t=1100 \mathrm{~ms}$, respectively. The arrows indicate the direction of cell movement. For the purpose of visualization, the "Main stream" blood vessel is highlighted by blurring the surrounding area of the stream. The movement of a cell in three dimensions in the stream was schematically illustrated in four dimensions by plotting the tracked blood cell along time axis with an interval of $100 \mathrm{~ms}$ (Video 6). Figure 5(d) and 5(f) show the relative locations [corresponding to Figs. 5(b) and 5(d), respectively] of the tracked cell in the "Main stream" in a 4-D representa- tion, the arrows also indicate the direction of the cell motion.

In the reconstruction processing, however, image quality was degraded due to the severe speckle observed. To eliminate speckle caused by the scattered light from the highly populated cells in the living tissue in Video 5, a motion detection technique, i.e., background subtraction, was utilized as a postreconstruction processing. The postreconstruction processing also includes a deshaking tool (Virtualdub 1.6.3). A deshaking processing is essential when a living tissue is chosen as the recording object because the bulk vibration of tissue was inevitable and it is impossible to obtain a "pure" background image for the processing. The resulting background-subtracted video frames (Video 7) were formed by subtracting frame $I(k)$ from $I(k+3)$, where, $k=1,2, \ldots$, $\mathrm{FN}-3$, and $\mathrm{FN}$ is the total number of frames in Video 5. For the purpose of visualization, a sum of all subtracted reconstruction images is calculated to show the blood stream tracks [Fig. 5(b)]. A moving blood cell in "Stream 2" is further 


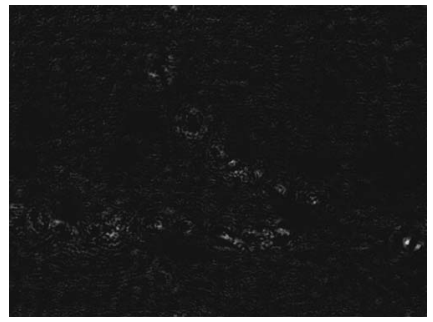

Video 7. Postreconstruction processing of the tadpole blood vessels eholovideo. A live tadpole was used to record a 3-s eholovideo (not shown) with a $10 \mathrm{fps}$ frame rate. The eholovideo was reconstructed (Video 5) along the time axis at the reconstruction distance $75 \mu \mathrm{m}$. Video 5 was then processed by a motion detection technique by a deshaking processing and subtracting the $k^{\prime}$ th frame from the $(k+3)^{\prime}$ th frame, $k=1,2, \ldots, \mathrm{FN}-3$, and $\mathrm{FN}$ is the total number of frames in Video 5 . The full frame size is 273 (width) $\times 204 \mu \mathrm{m}$ (height) (QuickTime 2.1 MB). [URL: http://dx.doi.org/10.1117/1.2841050.7]

traced from this processing and shown in Video 8, which lasts over $2400 \mathrm{~ms}$ along time axis with interval of $100 \mathrm{~ms}$. Video 8 shows cell movement at the actual recording rate of $10 \mathrm{fps}$ and at a $z$ distance of $49 \mu \mathrm{m}$. Some of the reconstructed frames are selected [Figs. $5(\mathrm{~g})-5(\mathrm{l})]$ from Video 8 to show the cell coordinators in four dimensions $\left(x^{\prime}, y^{\prime}, z, t\right)$. Therefore, the moving speed of the cell can be estimated by substituting the sensor pixel properties in $x^{\prime}$ and $y^{\prime}$. This individual blood cell in "Stream 2" flows with an estimated average speed $34 \mu \mathrm{m} \mathrm{s}^{-1}$, which is much slower than the cells in the "Main stream" (estimated average speed $195 \mu \mathrm{m} \mathrm{s}^{-1}$ ) and the cell size is measured approximately $2.8 \mu \mathrm{m}$ in diameter. An overall image resolution of around $1 \mu \mathrm{m}$ in this system is achieved.

\section{Conclusions}

In this paper, in vitro and in vivo fast-moving cells in a 3-D space were filmed nonintrusively using our DHVM system. The cell locations and 3-D spatial distributions of cells at one time point were recreated in a computer by scanning through the recorded volume at various depths for the chosen holo-

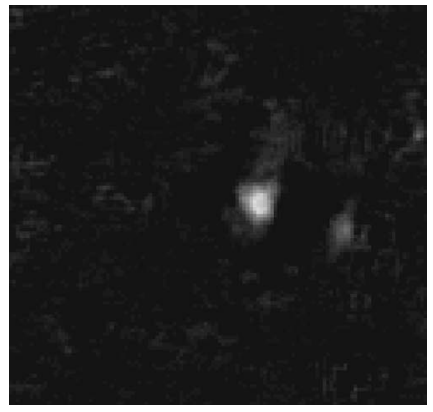

Video 8. Visualization of a single tadpole blood cell flowing in the blood stream in vivo. An individual blood cell in blood stream [marked as "Stream 2" in Fig. 5(a) in the main text] was recorded in a 3-s eholovideo (digital holographic video, not shown). The recorded eholovideo was then reconstructed along each time point (interval $100 \mathrm{~ms}$ ) and along $z$ to locate the best focusing plane. This video tracks the in-focus reconstructed cell over a 2400-ms time. The full frame size is 43 (width) $\times 41$ (height) $\mu \mathrm{m}$ (mov, $1.3 \mathrm{MB}$ ). [URL: http://dx.doi.org/10.1117/1.2841050.8] gram frame in the recorded eholovideo; while cell motions were recreated by reconstructing a sequence of eholovideo frames at a specific distance of cell focal planes in a time coordinate. In comparison with a conventional microscopic imaging system, an ehologram records a larger area and in a 3-D volume.

Recording fast-moving cells in suspension and in a blood stream in vivo has not been possible with currently available imaging systems because cells move in and out of the microscopic focal plane rapidly in a 3-D volume. With our digital video microscopic system, such rapid movements were recorded and recreated in a volumetric space. The DHVM system we report here is capable of recording video holographic images of cells on a glass slide, in a tissue culture dish, and in blood vessels. Both intensity and phase-contrast digital microscopic holograms of a 3-D object volume can be recorded. The recording of a nonstained live cell distribution in vivo and the movement of cells in a blood stream in vivo offers the exciting possibility of tracking fast cell movement in three dimensions. In comparison with other holographic systems, simplicity in video recording geometry is one of the main advantages of a DHVM system. Computer simulation of diffraction wave and video processing techniques are conveniently applied in the reconstruction of specimen eholovideos. The reference beam in reconstruction can be omitted if a parallel beam is used to illuminate the recording specimen. With selection of objective lenses, high spatial and temporal resolution can be achieved. The disadvantages of a DHVM include a very limited recording volume (several cubic centimeters) and restricted longitudinal image resolution when a highly magnifying lens is selected.

A limitation of our DHVM is the requirement of partial transparency of the specimen and the limited resolution for submicrometer recordings, where the scattering effect must be taken into account when carrying out size and distance measurements. When scattering becomes significant, the recorded hologram will be significantly degraded, to the point that no reference beam can reach the recording plane, in which case, no hologram will be formed and therefore no object image can be reconstructed by any of the mentioned reconstruction algorithms. This problem can be solved if an off-axis recording scheme, i.e., an angularly separated reference beam, is used. However, when cell population density is rather high, too much scattered light will produce severe speckle in a hologram due to the scattered coherence laser light from dense cells. Therefore, further developments on reconstruction algorithms and postreconstruction algorithms, recording geometry, and light sources are necessary.

\section{Acknowledgments}

We thank the funding bodies MRC (British Medical Research Council to HS, JW and MZ) and the Welcome Trust (to MZ). The work could not be started without the outcomes from a NERC (British National Environmental Research Council to JW and MAP) supported project (Biomediation of Sediment Erosion using laser holography). Thanks are also given to Drs. Stefan Hoppler and Fei Liu for their help with the tadpoles. 


\section{References}

1. E. Cukierman, R. Pankov, D. R. Stevens, and K. M. Yamada, "Taking cell-matrix adhesions to the third dimension," Science 294, 17081702 (2001).

2. B. Knight, C. Laukaitis, N. Akhtar, N. A. Hotchin, M. Edlund, and A. R. Horwitz, "Visualizing muscle cell migration in situ," Curr. Biol. 10, 576-585 (2000).

3. E. Sahai and C. J. Marshall, "Differing modes of tumour cell invasion have distinct requirements for Rho/ROCK signalling and extracellular proteolysis," Nat. Cell Biol. 5, 711-719 (2003).

4. D. J. Webb and A. F. Horwitz, "New dimensions in cell migration," Nat. Cell Biol. 5, 690-692 (2003).

5. M. Zhao, B. Song, J. Pu, J. V. Forrester, and C. D. McCaig, "Direct visualization of a stratified epithelium reveals that wounds heal by unified sliding of cell sheets," FASEB J. 17, 397-406 (2003).

6. M. Zhao, B. Song, J. Pu, T. Wada, B. Reid, G. Tai, F. Wang, A. Guo, P. Walczysko, Y. Gu, T. Sasaki, A. Suzuki, J. V. forrester, H. R. Bourne, P. N. devreotes, C. D. McCaig, and J. M. Penninger, "Electrical signals control wound healing through phosphatidylinositol3-OH kinase- $\gamma$ and PTEN," Nature (London) 442, 457-460 (2006).

7. H. Xu, A. Manivannan, D. Garry, J. Liversidge, P. F. Sharp, J. V. Forrester, and I. J. Crane, "Evaluation of leukocyte dynamic in mouse retinal circulation with scanning laser ophthalmoloscopy (Video report)," Br. J. Ophthamol. 85, 765-890 (2001).

8. E. Malkiel, J. Sheng, J. Katz, and J. R. Strickler, "The three dimensional flow field generated by a feeding calanoid copepod measured using digital holography," J. Exp. Biol. 206, 3657-3666 (2003).

9. G. Pan and H. Meng, "Digital holography of particle fields: reconstruction by use of complex amplitude," Appl. Opt. 42, 827-833 (2003).

10. U. Schnars and W. O. Jüptner, "Digital recording and numerical reconstruction of holograms," Meas. Sci. Technol. 13, R85-101 (2002).

11. J. H. Milgram and W. Li, "Computational reconstruction of images from holograms," Appl. Opt. 41, 853-864 (2002).

12. H. Sun, D. C. Hendry, M. A. Player, and J. Watson, "In situ underwater electronic holographic camera for studies of plankton," IEEE J. Ocean. Eng. 32(2), 1-10 (2007).

13. H. Sun, H. Dong, M. A. Player, J. Watson, D. M. Paterson, and R. G. Perkins, "In-line digital video holography for the study of erosion processes in sediments," Meas. Tech. 13, L7-12 (2002).

14. H. Sun, M. A. Player, J. Watson, D. C. Hendry, R. G. Perkins, G. Gust, and D. M. Paterson, "The use of digital/electronic holography for biological applications," J. Opt. A, Pure Appl. Opt. 7, S399-S407 (2005).

15. W. B. Xu, M. H. Jericho, I. A. Meinertzhagenm, and H. J. Kreuzer, "Digital in-line holography for biological applications," Proc. Natl. Acad. Sci. U.S.A. 98, 11301-11305 (2001).

16. L. Xu, X. Peng, J. Miao, and A. K. Asundi, "Studies of digital mi- croscopic holography with applications to microstructure testing,' Appl. Opt. 40, 5046-5051 (2001).

17. W. Xu, M. H. Jericho, H. J. Kreuzer, and I. A. Meinertzhagen, "Tracking particles in four dimensions with in-line holographic microscopy," Opt. Lett. 28, 164-166 (2003).

18. P. Marquet, B. Rappaz, and P. Magistretti, "Digital holographic microscopy: a non-invasive contrast imaging technique allowing quantitative visualization of living cells with subwavelength axial accuracy," Opt. Lett. 30, 468-470 (2005).

19. F. Dubois, N. Callens, C. Yourassowsdy, M. Hoyos, P. Kurowski, and O. Monnom, "Digital holographic microscopy with reduced spatial coherence for three-dimensional particle flow analysis," Appl. Opt. 45, 846-870 (2006).

20. C. J. Mann, L. Yu, and M. K. Kim, "Movies of cellular and subcellular motion by digital holographic microscopy," Biomed. Eng. Online 5(21), 5:21 doi:10.1186/1475-925X-5-21 (2006).

21. J. Sheng, E. Malkiel, and J. Katz, "Digital holographic microscope for measuring three-dimensional particle distributions and motions," Appl. Opt. 45, 3893-3901 (2006).

22. U. Schnars and W. O. Jüptner, "Direct recording of holograms by a CCD target and numerical reconstruction," Appl. Opt. 33, 2187-2197 (1994).

23. R. B. Owen and A. A. Zozulya, "In-line digital holographic sensor for monitoring and characterizing marine particulates," Opt. Eng. 39, 2187-2197 (2000).

24. U. Schnars and W. O. Jüptner, "Digital recording and numerical reconstruction of holograms," Meas. Sci. Technol. 13, R85-101 (2002).

25. X. M. Huang, J. M. Zuo, and J. H. Spence, "Wavefront reconstruction for in-line holograms formed by pure amplitude objects," Appl. Surf. Sci. 148, 229-234 (1999).

26. D. Lebrun, S. Belaid, and C. Ozkul, "Hologram reconstruction by use of optical wavelet transform," Appl. Opt. 38, 3730-374 (1999).

27. M. Liebling, T. Blu, and M. Unser, "New multiresolution wavelet bases for digital holography," IEEE Trans. Image Process. 12, 29-43 (2003).

28. D. C. Champney, Fourier Transforms and Their Physical Applications, pp. 138-180, Academic Press, London (1973).

29. C. Buraga-Lefebvre, S. Coëtmellec, D. Lebrun, and C. Özkul, "Application of wavelet transform to hologram analysis: threedimensional location of particles," Opt. Lasers Eng. 33, 409-421 (2000).

30. L. Onural and M. Kocatepe, "Family of scaling chirp functions, diffraction, and holography," IEEE Trans. Signal Process. 43, 15681578 (1995).

31. H. Dong, C. Khong, M. A. Player, M. Solan, and J. Watson, "Algorithms and applications for electronically-recorded holography," in Proc. 6th Int. Conf. on Correlation Optics, O. V. Angelsky, Ed., Proc. SPIE, 5477, 354-365 (2004). 